\title{
Realization of Low Confinement Loss Acetylene Gas Sensor by Using Hollow-core Photonic Bandgap Fiber
}

\section{Hassan Arman}

Shahid Rajaee Teacher Training University

Saeed Olyaee ( $\nabla$ s_olyaee@sru.ac.ir)

Nano-photonics and Optoelectronics Research Laboratory (NORLab), Faculty of Electrical Engineering, Shahid Rajaee Teacher Training University (SRTTU), Lavizan, Tehran, Iran https://orcid.org/0000-00026154-7646

\section{Original Research}

Keywords: Confinement loss, hollow-core PBF, gas sensing, interference suppression, acetylene

Posted Date: February 4th, 2021

DOl: https://doi.org/10.21203/rs.3.rs-175335/v1

License: (c) (i) This work is licensed under a Creative Commons Attribution 4.0 International License. Read Full License 


\title{
Realization of low confinement loss acetylene gas sensor by using hollow-core photonic bandgap fiber
}

\author{
Hassan Arman ${ }^{1}$ and Saeed Olyaee ${ }^{2, *}$ \\ ${ }^{1}$ Faculty of Electrical Engineering, Shahid Rajaee Teacher Training University (SRTTU), 16788-15811, Tehran, Iran \\ 2 Nano-photonics and Optoelectronics Research Laboratory (NORLab), Faculty of Electrical Engineering, Shahid Rajaee \\ Teacher Training University (SRTTU), Lavizan, Tehran, Iran \\ *Email: s_olyaee@sru.ac.ir
}

\begin{abstract}
:
A hollow-core photonic bandgap fiber (HC-PBF) with high relative sensitivity and low confinement loss was designed. Some destructive circumstance such as propagation losses and mode interference can disrupt performance of the PBF. By considering optimum size of the hollow-core radius, we were able to improve confinement loss and the relative sensitivity. By optimization of the shape and size of the closest row of air holes to the hollow core, the quality of the mode distribution in the hollowcore was well improved. Simulation results confirm that, at an optimal and reasonable core radius, the relative sensitivity and confinement loss of the proposed gas sensor were improved to $96.5 \%$ and $0.11 \mathrm{~dB} / \mathrm{m}$, respectively. In addition, in order to better matching of optical power between single mode fiber (SMF) and HC-PBF, we could reduce the destructive effects of optical mode mismatch, by mode interference suppression. Furthermore, by optimization of fiber structural specifications such as air filling fraction and lattice constant, the PBF was changed to a single-mode waveguide. Considering the operation wavelength $1530 \mathrm{~nm}$ which is very close to the acetylene gas absorption wavelength, this fiber is appropriate to be a high sensitivity gas sensor to detect absorbing gases in the middle infrared range.
\end{abstract}

Keywords: Confinement loss; hollow-core PBF; gas sensing; interference suppression; acetylene.

\section{Introduction}

Recent advances in fiber optics and related equipment have led to significant advances in the development of optical sensor technology. Gas sensors based on photonic crystal fibers have special features that some of them are: small dimension, low price, ability to work in high temperature environments, wide bandwidth, and more accuracy in measurement [1]-[7]. Features such as resistance to harsh environments and immunity to the electromagnetic interference, offer appropriate options for a wide range of sensing applications and their superiority in the use of evaluation of different parameters make them more susceptible to other equivalent sensors. Moreover, they have the potential to convert to a well-set device that can perform remote sensing [8].

A novel and remarkable kind of fiber optic waveguides that a lot of attention has been paid to it, is hollow core photonic bandgap fiber (HC-PBF). The photonic bandgap fibers provide unique optical properties that could not be achieved in conventional photonic crystal fibers such as index guiding, 
high birefringence and high nonlinearity, to name a few [5]-[9]. In this kind of fibers, more optical power was confined in the hollow-core. Hollow core photonic bandgap fibers are particularly useful in sensing technology because they can provide a long interaction length in a compact area. The sensitivity of gas sensors based on this type of fiber, is typically high [9]-[11]. In other words, due to one of the excellent advantages of hollow core photonic bandgap fiber, which is significant overlap between light and hollow waveguides especially the overlap between optical mode and hollow-core, they are considered a suitable alternative to such sensors [12], [13]. However, developing a gas sensor with such distinguished features is not free of challenges. Due to the large core, they are highly multi-mode, which could create an issue for modal unification. In addition to the fundamental mode, higher order modes will also be present in the large hollow core [8]. Surface modes can also interfere with the fundamental mode and greatly disrupt sensor performance [9], [13].

So far, many researchers have studied to use the photonic bandgap fibers (PBFs) as a gas sensor and various methods were proposed. Recently, studies have been conducted on gas sensors such as surface plasmon resonance-based gas sensor and toxic gas detection [14], [15]. Each of the gas sensors we've talked about so far, has been able to improve the performance of hollow core fibers in gas detection [9]-[15].

Gases, in general, present absorption spectra, which depend on their molecular structure and is unique to each gas. The absorption wavelength of number of commonly used gases such as acetylene $\left(\mathrm{C}_{2} \mathrm{H}_{2}\right)$ and methane $\left(\mathrm{CH}_{4}\right)$ are within near infrared range [16]. Hence, many papers have been reported on gas sensors in this specific wavelength range [17]-[20]. Each of them has demonstrated many configurations using photonic bandgap fiber for gas detection. An investigation into the feasibility of using HC-PBF in methane gas detection in the $1.3 \mu \mathrm{m}$ region was reported by Cubillas et al. [21]. Ritari et al., described a micro structured fiber in acetylene gas detection in the $1.5 \mu \mathrm{m}$ wavelength [22]. Similarly, a lot of approaches have also been implemented.

Optical absorption, due to gas molecular transition, is a linear and simple optical property that can be easily measured [16]. The absorption-based mechanism is used in many photonic crystal fiberbased gas sensors. This mechanism can be interpreted as follows: the interaction of optical mode and analyte along the hollow core reduces optical power due to absorption. The analyte can be monitored by calculating the amount of optical power lost due to absorption [16]-[20].

As mentioned above, almost all of the light power is located in the hollow-core of the photonic bandgap fiber. However, there are destructive parameters that limit the performance of PBF-based gas sensors; presence of unstable guided higher order modes and the mismatch of light coupling that cause mode interference and mode mismatch, respectively [12], [23]. Photonic band gap fibers depending on the dimensions and the shape of the hollow-core, may support more than one mode. This means the hollow core radius will have a large effect on the number of supported optical modes. Meanwhile surface modes are one of the effective factors on the performance of photonic bandgap fibers are specified by the thickness of the silica layer that surround the hollow core. [13]. Light coupling discrepancy between the SMF and the HC-PBF will deteriorate the performance of the gas sensor based on hollow-core photonic bandgap fiber [9] so, an appropriate method should be considered. The relative sensitivity and confinement loss are two important propagation specifications of the PBF based gas sensors which are highly dependent on the structural characteristics of the fiber [23], [24]. Recently, we proposed a HC-PBF based gas sensor [23]. The sensitivity of this sensor was improved by optimization of core size and mode interference suppression.

In this paper, an acetylene gas sensor based on hollow-core photonic bandgap fiber was presented. We have demonstrated reasonable size of the hollow-core radius and mode interference elimination, play an important role in improving the relative sensitivity and reduce the confinement loss of the designed gas sensor. By applying accurate changes to the radius of the hollow core and optimization of the shape and size of the closest row of air holes to the hollow core we have improved 
the performance of the proposed sensor. In fact, the high relative sensitivity and low confinement loss of proposed sensor led to excellent performance for gas detection in the field of environmental and industrial surveillance.

\section{Gas sensing mechanism}

The setup used to investigate the gas sensing mechanism is illustrated in Fig. 1. Here, the optical power with a proper wavelength from an adjustable light source (TLS) was emitted inside the HCPBF through a single-mode fiber (SMF-28). Simultaneously, the gas sample with an absorption wavelength close to the wavelength of the incoming light source was launched into the HC-PBF. The end edge of the HC-PBF was coupled to the second SMF which transmit the output light to the photodetector (PD).

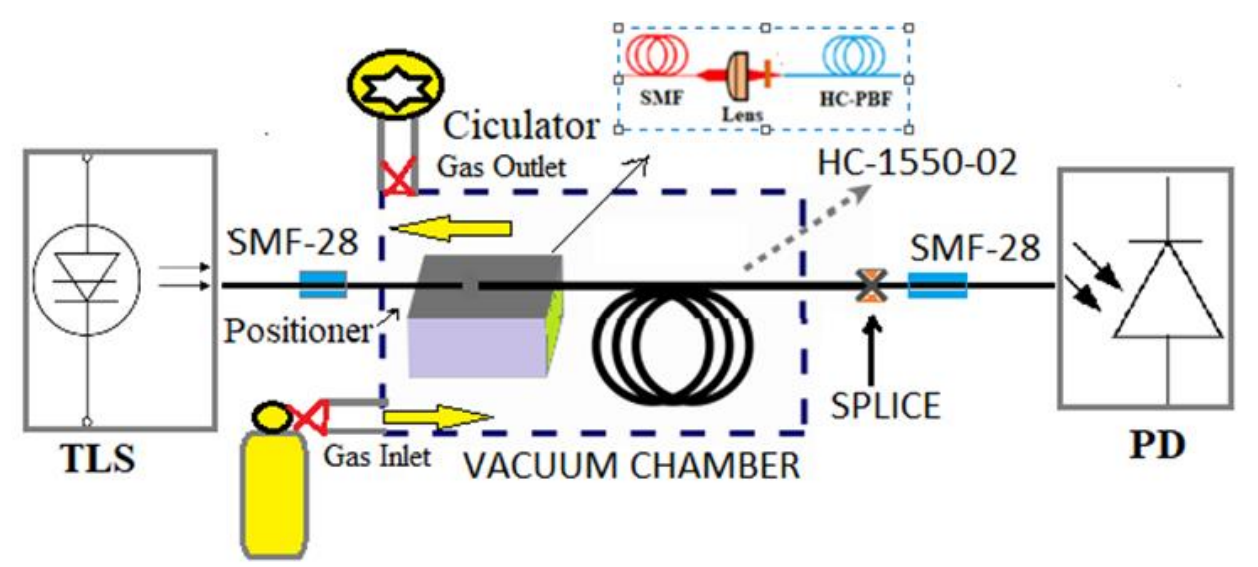

Fig. 1: The schematic diagram of the of the proposed HC-PBGF based gas sensor.

The middle part of this structure, the hollow core of the PBF, acts as a waveguide, where due to the high overlap, light and gas samples interact along the hollow core and gas sensing is performed. In other words, the light and gas sample interact inside the hollow core and due to absorption, some of the transmitted optical power is attenuated according to the Beer-Lambert equation as [12]:

$$
I(\lambda)=I_{0}(\lambda) \exp \left[-r \alpha_{m}(\lambda) L C\right]
$$

where, $\alpha_{m}(\lambda)$ is the absorption coefficient of the gas sample, $I$ and $I_{0}$ are the output light intensities with and without the existence of the tested gas, respectively. Also, the gas concentration and the length of the waveguide are respectively denoted by $C$ and $L$. The relative sensitivity coefficient $(r)$ which is directly related to the percentage of optical power that entered into the hollow-core can be calculated by $r=\left[n_{r} / n_{\text {eff }}\right] f$ where, $n_{r}$ refers the refractive index of the gas sample which in calculations is usually assumed to be unit. The effective refractive index of the fundamental mode is determined by $n_{\text {eff }}$. The amount of the total optical power confined in the hollow-core is shown by $f$ that can be estimated by [23], [25]:

$$
f=\frac{\int_{\text {hollow core }}\left(\mathrm{E}_{\mathrm{x}} \mathrm{H}_{\mathrm{y}}-\mathrm{E}_{\mathrm{y}} \mathrm{H}_{\mathrm{x}}\right) \mathrm{dxdy}}{\int_{\text {total }}\left(\mathrm{E}_{\mathrm{x}} \mathrm{H}_{\mathrm{y}}-\mathrm{E}_{\mathrm{y}} \mathrm{H}_{\mathrm{x}}\right) \mathrm{dxdy}}
$$

where, $H_{x}, H_{y}$ and $E_{x}, E_{y}$ are the transverse magnetic and electric fields of the dominant optical mode, respectively. 
The confinement loss of the PBF can directly be estimated by imaginary part of the effective refractive index using the finite element method (FEM). The confinement loss $L_{c}$, in decibel per meter $(\mathrm{dB} / \mathrm{m})$ can be calculated by [26], [27]:

$$
\mathrm{L}_{\mathrm{c}}=(17.37 \pi / \lambda) \operatorname{Im}\left[n_{\mathrm{eff}}\right]
$$

where, $\operatorname{Im}\left[n_{\text {eff }}\right]$ is the imaginary part of effective refractive index of fundamental mode, respectively.

Since almost all HC-PBFs support several guided modes, especially when their hollow core is considered larger, the interference between the guided modes leads to changes in the intensity of the transmitted light [20]. Since HC-PBF -based gas sensors that work with the optical power absorption mechanism are highly dependent on light intensity, their relative sensitivity is also expected to be highly dependent on changes in light intensity. Thus, eliminating the mode interference will increase the relative sensitivity [13], [28]. The first step is to pass light through a single-mode fiber before being directed into the photonic bandgap fiber, so mode mismatch at the junction of the two mentioned fibers can be suppressed. One of effective methods to do this is to use a spherical lens [16]. As depicted in Fig. 1, two aspheric lenses were incorporated, one of them is placed before and the other one after the HC-PBF to make the output beam between the SMF and HC-PBF more cohesive and focused. In fact, lenses were employed to improve mode matching and to enhance the fundamental mode stimulation and minimize the confinement loss in the HC-PBF. The distribution of the normalized electric field at the junction of the single-mode fiber and the photonic band gap fiber is depicted in Fig. 2.

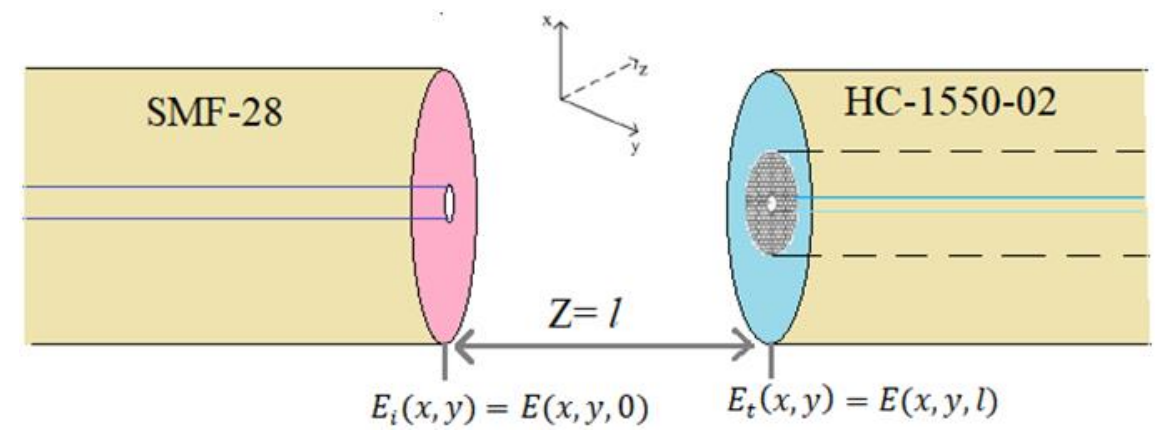

Fig. 2: Schematic of the optical mode excitation from SMF-28 to HC-1550-02 fiber.

According to Fig. 2, the normalized field distribution of the single-mode fiber can be explained by a Gaussian relation if we assume that the polarization of the electric field is in the direction of the $\mathrm{x}$ axis [13] $\left.E(x, y)=E_{0} \exp \left(-\left(x^{2}+y^{2}\right) / w^{2}\right)\right)$ where, $w$ and $E_{0}$ refer the mode field radius and the electric field amplitude of the beam center of the SMF, respectively. If we show the waist radius of the Gaussian light beam that is focused using the lenses with $w$, then the waist radius of the output light beam will be shown by the following equation [29]:

$$
W_{0}=\sqrt{\frac{F^{2} W^{2}}{(F-l)^{2}+\left(\frac{\pi w^{2}}{\lambda}\right)}}
$$

where, $\lambda$ is the operation wavelength, $F$ is the effective focal length of the lens, and $l$ is the longitudinal distance between the incident light beam waist and each of the lenses. Also, the electric field will be distributed at the input edge of the HC-PBF by: 


$$
E_{i}(x, y)=E_{1} e^{-\frac{x^{2}+y^{2}}{w_{1}^{2}}}
$$

where, $E_{1}$ is the amplitude of electric field and $w_{1}$ is the waist radius at the input edge of the HC-PBF. The distribution of the total transverse electric field $E_{\mathrm{t}}$, at the output edge of the HC-PBF can be described by [17], [30]:

$$
E_{t}(x, y) \cong E_{i}(x, y) \frac{2 n_{\mathrm{air}}}{n_{\mathrm{air}}+n_{\mathrm{HC}}}
$$

where, $n_{\text {air }}$ and $n_{H C}$ are the refractive indices of air and hollow-core, respectively. It can be assumed $n_{\text {air }} \cong n_{\mathrm{HC}}$ so Eq. (5) can be simply rewritten as $E_{\mathrm{t}}(x, y) \cong E_{\mathrm{i}}(x, y)$.

From recent discussions, it is understood that the coupling efficiency of the fundamental modes between the single-mode fiber and the hollow core fiber is acceptable when we can obtain the modal field distribution in the hollow core fiber as close as possible to the diameter of the input beam. In the next step, we intend simulate the parameters that mentioned above and discussed and examine them numerically.

\section{Results of mode interference elimination}

In order to evaluate mode matching at the junction of the SMF and the HC-PBF, a corning SMF-28 and a NKT Photonics, HC-1550-02 were considered [14], [15]. According to the structural properties of both fibers, the dimensions of each fibers were considered as follows: the core radius and pitch of the HC-PBF are $5.82 \mu \mathrm{m}$ and $3.88 \mu \mathrm{m}$, respectively, and the core and cladding radius of SMF are 4.1 $\mu \mathrm{m}$ and $62.5 \mu \mathrm{m}$, respectively. Due to the high accuracy of the simulation, COMOSL Multiphysics software was used to evaluation the waist diameter of the SMF mode field and the fundamental mode field of the HC-PBF. The fundamental mode field distribution of both fibers at the operation wavelength was clearly shown in Fig. 3.

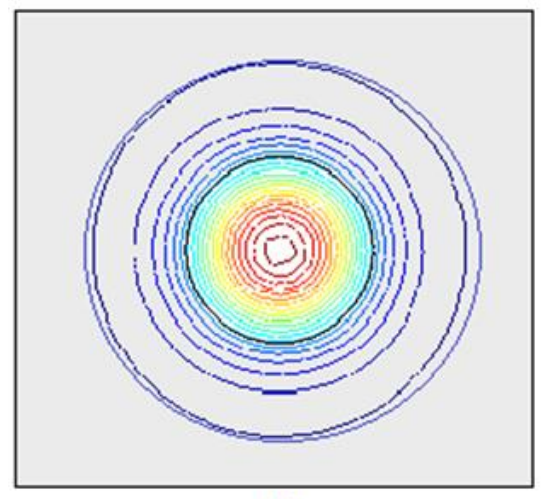

(a)

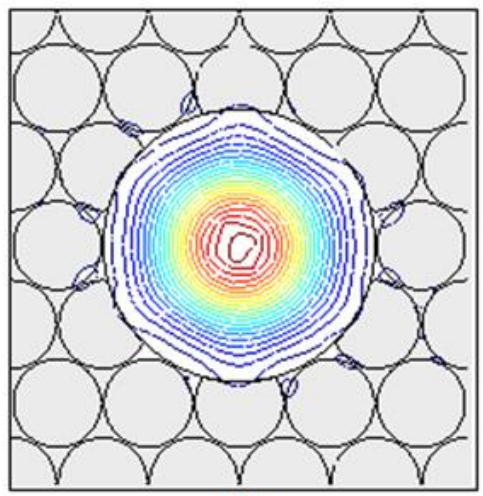

(b)

Fig. 3: The representation of fundamental mode distribution for (a) single mode fiber and (b) hollow-core photonic bandgap fiber.

According to Fig. 3, although the core radius in either of fibers is not equal but due to the placement of the lenses between them, their beam diameters are very close to each other. The normalized electric field intensity which is distributed in Gaussian form versus core radius changes, numerically examined as illustrated in Fig. 4. The radius of the optical mode field of SMF-28 and the radius of the fundamental mode field of HC-1550-02 can be evaluated. The amounts of full-width at half-maximum (FWHM) were respectively obtained as $6.5 \mu \mathrm{m}$ and $6.2 \mu \mathrm{m}$ for single mode fiber and hollow-core photonic bandgap fiber. The aspheric lenses were employed to improve mode matching 
between two fibers [15], [31]. A similar mechanism is performed at the output of HC-PBF by the second lens, and finally, the output light is directed to the photodetector.

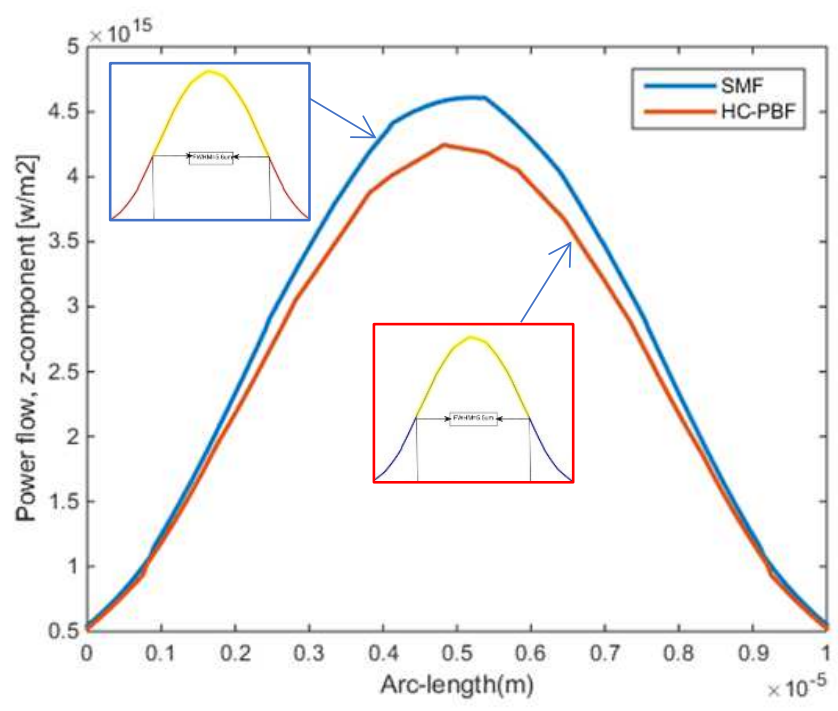

Fig. 4: The normalized electric field intensities versus the core radius.

\section{Results of confinement loss reduction and relative sensitivity improvement}

A proposed setup was designed for gas sensing which was illustrated in the previous section. In this section, we have studied the magnitude of optical power that is trapped inside the hollow waveguide. In order to obtain the best design, we analyze the structural parameters and optical specifications of the proposed setup, using one of the most accurate and reliable numerical methods for analyzing photonic bandgap fibers. This numerical method is finite-element method (FEM) which is the most suitable method for analyzing the structural parameters of photonic crystal fibers. Optimization of the hollow-core radius an essential action for reducing confinement losses and consequently enhanced the relative sensitivity [23],[26].

By carefully selecting the effective radius of the hollow core, we were able to improve the relative sensitivity and confinement losses of the proposed gas sensor. In fact, according to the Beer-Lambert law, some of the guided light is absorbed by the gas sample, so some of the optical power is attenuated. Therefore, the gas within the HC-PBGF can be monitored by estimate the attenuation of the guided light in the waveguide. In order to estimate the relative sensitivity of the proposed gas sensor, different values for the hollow-core radius were considered to obtain the most appropriate value. So, we calculated the relative sensitivity in a desired radial range, by accumulation the optical power located in the hollow-core, and dividing it by the total optical power carried by the fundamental mode [9], [28].

Because in this design, the fiber guides the light with the photonic band gap mechanism, only particular light wavelengths will be allowed to propagate inside the waveguide [18], [32]. On the other hand, for the best sensor performance, the absorption line of the gas sample to be detected must be within the photonic bandgap. Since the structural characteristics of the HC-PBF was designed for acetylene gas sensor, it is essential to consider the operation wavelength very close to the gas absorption line. The absorption line of acetylene gas is approximately $\lambda_{\text {absorb }}=1.53 \mu \mathrm{m}$ [7], So, 
considering the bandgap region, we select the operation wavelength $\lambda_{\text {operation }}=1.55 \mu \mathrm{m}$. Therefore, in our proposed design, the structural parameters of the hollow core fiber were considered as follows ; $\Lambda=3.88 \mu \mathrm{m}, \frac{\boldsymbol{d}}{\Lambda}=0.97$, where $\Lambda$ is the lattice constant and $d$ is the diameter of the air holes of the cladding.

In all analyses, according to the structural specifications of the HC-PBF-1550-2, the air holes indices and the silica refractive index were considered to be equal to 1 and 1.45, respectively [33], [34]. In order to locating the operation wavelength inside the bandgap, the structural parameters were carefully considered. So, the structural specifications were considered as follows: the air-filling factor of 0.97, and the lattice constant of $\Lambda=3.88 \mu \mathrm{m}$ [7], [23].

The proposed fiber cross-section including the air holes and the hollow core is clearly shown in Fig. 5. As depicted in Fig. 5, the HC-PBF comprises of a hexagonal lattice of air holes that the hollowcore was formed by removing two rows of air holes from center of the structure.

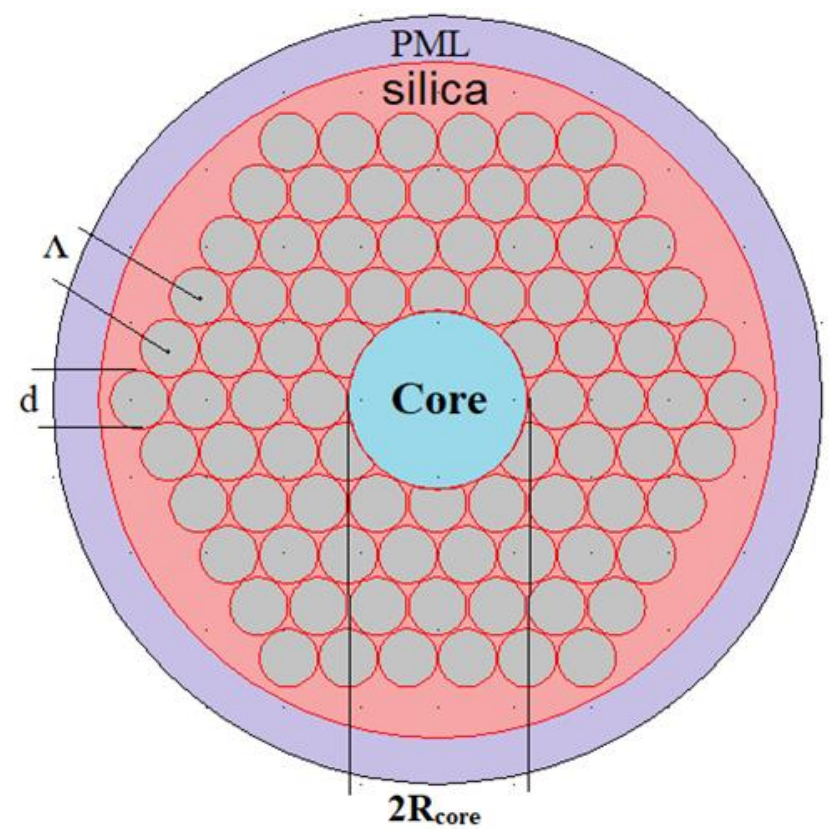

Fig. 5: The schematic diagram of the hollow-core photonic bandgap fiber (NKT Photonics, HC-155002).

Two parameters, each of which will play a significant role in the efficiency of the proposed gas sensor are surface modes and higher order modes. These destructive modes can be paired with the fundamental mode and deteriorated the performance of PBF. [7], [9]. So, one of the important parameters which must be considered is the selecting of the hollow core radius, which determines the quality and effect of mode interference. Surface modes are detected when the part of the substrate that is close to the fiber core was abruptly interrupted [7], [13]. In other words, surface modes appear when the side surfaces of the hollow-core intersect one or more of the dielectric corners of the photonic crystal substrate. To eliminate the effects of the surface modes, the portions of the hollow core must pass through the parts with different dielectric coefficients from the substrate to reduce its intersection with dielectric zones as much as possible [24], [35]. Calculating the confinement loss of the HC-PBF can be a suitable criterion for the impact of destructive factors such as surface mode, 
mode interference, and higher order modes. As stated earlier the confinement loss of the proposed HC-PBF can be easily obtained by multiplication of wavelength and imaginary part of the effective refractive index that obtained in FEM analysis [36], [37].

To investigate this supposition, the confinement loss in the mentioned radial range were examined, and the results are depicted in Fig. 6. As clearly shown, the confinement loss per radius $5.5 \mu \mathrm{m}$ and $5.8 \mu \mathrm{m}$ is minimum. It is clear that by minimizing the confinement loss, we can expect the relative sensitivity of the designed gas sensor to be greatly enhanced. To prove this assumption, we examined the percentage of relative sensitivity for the mentioned radial range. So, to achieve the desired radius, the relative sensitivity in the radial range from $1.35 \Lambda$ to $1.5 \Lambda$ in the step of $0.3 \Lambda$ was calculated as depicted in Fig. 6 (the hollow core radius was incremented by $120 \mathrm{~nm}$, at each step).

The relative sensitivity was examined by accumulation the optical power in the hollow-core and dividing it by total power carried by the fundamental mode in the whole proposed fiber structure. According to Eq. (2), the relative sensitivity is directly proportional to the fraction of the optical power that limited in the hollow-core.

To find the most suitable radius of the hollow core by using the FEM, we obtained the relative sensitivity for a desired radial range. According to Fig. 6, considering the hollow-core radius, $\mathrm{R}=5.8$ $\mu \mathrm{m}$; the relative sensitivity was improved to 96.55 . This improvement could be due to the consideration of large hollow core which led to the limitation of more optical power in the hollow core. But the interesting point that is also illustrated in this figure, is that the maximum relative sensitivity occurred in a negligible confinement loss which this proves the functionality of the prosed gas sensor more than before.

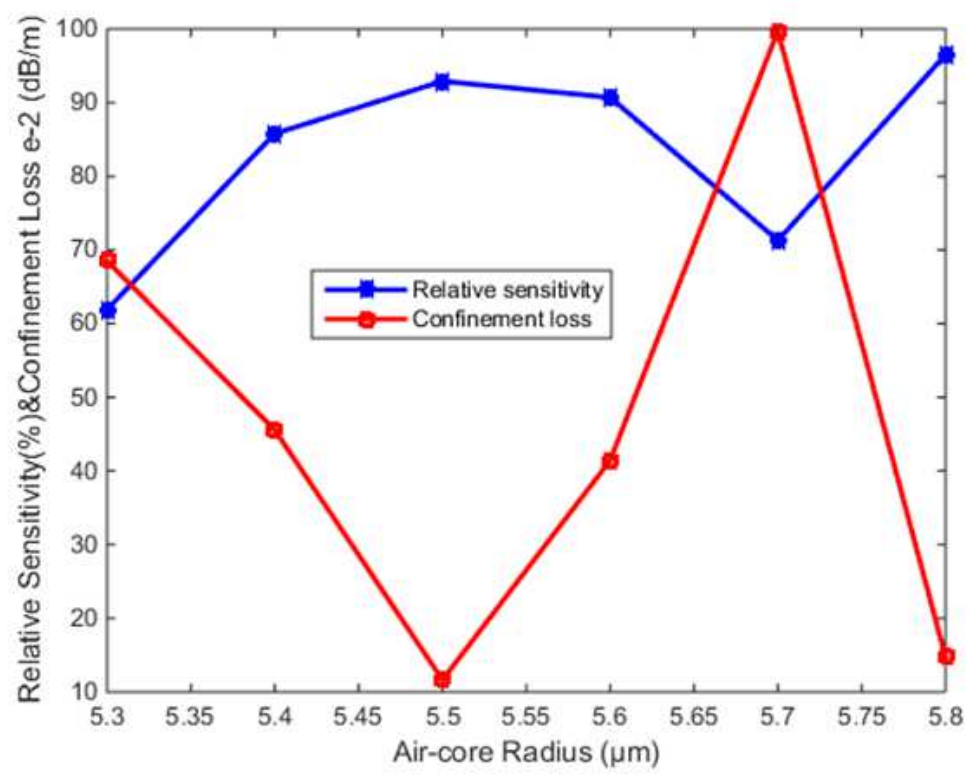

Fig. 6: The relative sensitivity and confinement loss versus hollow-core radius.

Another reason that further confirms this result is the study of the optical mode distribution in the hollow core for different radii [23]. In order to investigate the effect of the hollow core radius variations on the relative sensitivity, the quality of fundamental mode distribution in the hollow core in the form of a gaussian diagram are compared in Fig. 7. The radius of the hollow core with the same recent steps is changed. The most appropriate mode distribution appears in the radius at which the relative sensitivity is highest. This result can be interpreted as follows: the minimum amount of 
confinement loss in the mentioned radius has led to better light trapping in the hollow core and consequently has optimized the fundamental mode distribution.

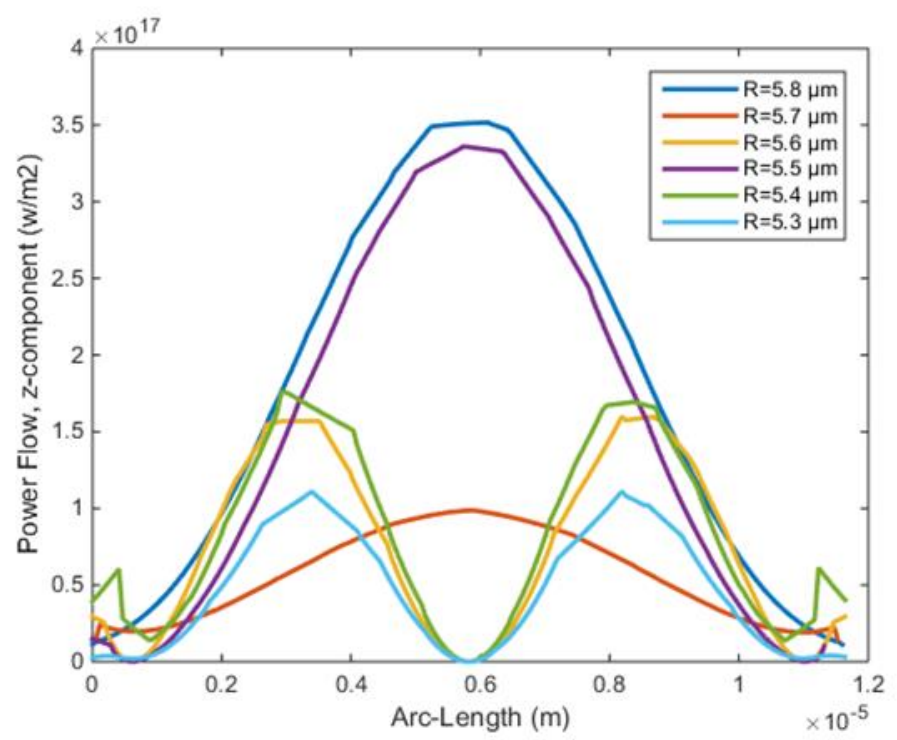

Fig. 7: The relative sensitivity and confinement loss versus hollow-core radius.

From all recent discussions, it can be concluded that the effect of destructive factors on the performance of the proposed gas sensor was studied. By applying effective solutions to the proposed structure, high relative sensitivity and negligible confinement loss in an optimal core radius were simultaneously obtained.

\section{Conclusion}

We have proved that mode interference elimination and considering a well-founded size of the fiber hollow-core radius can simultaneously improve the confinement loss and relative sensitivity of the proposed structure for gas sensor. We studied the effect of mode interference and structural parameters on the performance of HC-PBF based gas sensor. The destructive effects of mode interference were studied and appropriate techniques were proposed to overcome these factors. The use of spherical lenses for better focus of incoming light as well as the selection of an optimal core radius led to an improvement in the relative sensitivity of the proposed sensor and minimization of confinement loss. In fact, the result of using an exact radius was that we achieved a negligible confinement loss and high relative sensitivity with suppressed mode interference. Combination of solutions mentioned above are being considered that led to a $0.11 \frac{\mathrm{dB}}{\mathrm{m}}$ confinement loss and $96.5 \%$ relative sensitivity for gas sensor.

\section{Declarations section}

\section{Competing Interest}

The authors declare that they have no competing interests.

\section{Author's Contributions}


HA designed and simulated the sensor, and drafted the manuscript. SO supervised, reviewed, and edited the manuscript. All authors read and approved the final manuscript.

\section{Funding}

This work was supported by Shahid Rajaee Teacher Training University (SRTTU).

\section{Acknowledgements}

This research has been done in Nano-photonics and Optoelectronics Research Laboratory (NORLab) and the authors would like to thank Shahid Rajaee Teacher Training University for supporting of this research project.

\section{References}

[1] V. Portosi, D. Laneve, M.C. Falconi and F. Prudenzano, "Advances on Photonic Crystal Fiber Sensors and Applications”, Sensors, Vol. 19, No. 8, pp.1-19, 2019.

[2] B. Painam, R.S. Kaler and M. Kumar, "Photonic Crystal Waveguide Biochemical Sensor for the Approximation of Chemical Components Concentrations", Plasmonics, Vol. 12, No. 3, pp. 899-904, 2017.

[3] M. De, T.K. Gangopadhyay and V.K. Singh, "Prospects of Photonic Crystal Fiber as Physical Sensor: An Overview”, Mdpi Sensors, Vol.19, No. 3, pp.1-27, 2019.

[4] S. Olyaee and A. Naraghi, "Design and optimization of index-guiding photonic crystal fiber gas sensor", Photonic Sensors, Vol. 3, No. 2, pp. 131-136, 2013.

[5] P. Lova, G. Manfredi, C. Bastianini, C. Mennucci, F. Buatier de Mongeot, A. Servida and D. Comoretto, "Flory-Huggins Photonic Sensors for the Optical Assessment of Molecular Diffusion Coefficients in Polymers", ACS Applied Material, Vol. 11, No. 13, pp. 16872-16880, 2019.

[6] J. Park, S. Lee, S. Kim, and K. Oh, "Enhancement of chemical sensing capability in a photonic crystal fiber with a hollow high index ring defect at the center", Opt. Exp., vol. 19(3), pp. 1921-1929, 2011.

[7] S. Olyaee, H. Arman and A. Naraghi, "Design, Simulation, and Optimization of Acetylene Gas Sensor Using Hollow-Core Photonic Bandgap Fiber", Sensor Letters, Vol. 13, pp 1-6, 2015.

[8] Z. A. Zaky, A. M. Ahmed, A. S. Shalaby and A. H. Aly, "Refractive index gas sensor based on the Tamm state in a onedimensional photonic crystal: Theoretical optimization". Scientific Reports, Vol. 10, pp. 19, 2020.

[9] H. Arman, S. Olyaee and A. Mohebzadeh, "Gas Sensor Based on Large Hollow-Core Photonic Bandgap Fiber," International Journal of Optics and Photonics (IJOP), Vol. 9, No. 2, pp. 99-106, 2015.

[10] T. Ritari, J. Tuminen, and H. Ludvigsen "Gas sensing using air-guiding photonic bandgap fiber," Opt. Express, vol. 12, pp. 4081-4087, 2004.

[11] R. Thapa, K. Knabe, M. Faheem, A. Naweed, O. L. Weaver, and K. L. Corwin, "Saturated absorption spectroscopy of acetylene gas inside large-core photonic bandgap fiber," Opt. Lett. vol. 31, pp. 2489-2491, 2006.

[12] Y.L. Hoo, W. Jin, H.L. Hoo, J. Ju, and D.N. Wang, "Gas diffusion measurement using hollow-core photonic bandgap fiber," Sensors Actuat. B-Chem. vol. 105, pp. 183-186, 2004.

[13] K. Saitoh, N.J. Florous, T. Murao, and M. Koshiba, "Realistic design of large-hollow core photonic band-gap fibers with suppressed higher order modes and surface modes", Light Technol., vol. 25, pp. 2440-2447, 2007. 
[14] M. Ghodrati, A. Farmani and A. Mir, "Nanoscale Sensor-based Tunneling Carbon Nanotube Transistor for Toxic Gases Detection: A First-Principle Study", IEEE Sensor Journal, vol. 19, pp. 7373-7378, 2019.

[15] N. Amoosoltani, A. Zarifkar and A. Farmani, "Particle swarm optimization and finite-difference time-domain (PSO/FDTD) algorithms for a surface plasmon resonance-based gas sensor", Journal of Computational Electronics, vol. 18, pp. 1354-1364, 2019.

[16] E. Austin, A. van Brakel, M. N. Petrovich, and D. J. Richardson, "Fibre optical sensor for C2 H2 gas using gas-filled photonic bandgap fibre reference cell," Sensors and Actuators B: Chemical, vol. 139, pp. 30-34, 2009.

[17] L. Hu et al, "A hollow-core photonic band-gap fiber based methane sensor system capable of reduced mode interference noise", Infrared Physics and Technology, vol. 97, pp. 101-107, 2019.

[18] M. Ibadul Islama et.al, "Design of single mode spiral photonic crystal fiber for gas sensing applications", Sensing and Bio-Sensing Research, vol. 13 pp. 55-62, 2017.

[19] Y. L. Hoo, W. Jin, H. L. Ho, and D. N. Wang. "Measurement of gas diffusion coefficient using photonic crystal fiber," IEEE Photon Technol, vol. 15, pp 1434-1436, 2003.

[20] S.M.M. Quintero, L.C.G. Valente, M.S.P. Gomes, H.G. Silva, B.C. Souza, S.R.K. Morikawa, “All-Fiber CO2 sensor using hollow core PCF operating in the $2 \mu \mathrm{m}$ Region", Journal of Sensors, vol. 18, No. 12, pp. 1-10, 2018.

[21] A.M. Cubillas, J.M. Lazaro, M. Silva, O.M. Conde, M.N. Petrovich and J.M. LOPEZ, "Methane sensing at $1300 \mathrm{~nm}$ band with hollow-core photonic bandgap fibre as gas cell", Electron. Letter, Vol. 44, pp. 403-404, 2008.

[22] T. Ritari, "Gas sensing using air-guiding photonic bandgap fiber”, Opt. Express, Vol. 12, pp. 40804087, 2004.

[23] H. Arman and S. Olyaee, "Improving the sensitivity of the HC-PBF based gas sensor by optimization of core size and mod interference", Optical and Quantum Electronic, Vol. 52, No. 418, pp. 1-10, 2020.

[24] S. Chowdhury et.al, "Porous shaped photonic crystal fiber with strong confinement field in sensing applications: Design and analysis", Sensing and Bio-Sensing Research, vol. 13 pp. 63-69, 2017.

[25] S. Asaduzzaman, and K. Ahmed, "Proposal of a gas sensor with high sensitivity, birefringence and nonlinearity for air pollution monitoring," Sensing and Bio-Sensing Research, vol. 10, pp. 2026, 2016.

[26] K. Saitoh and M. Koshiba, "Leakage loss and group velocity dispersion in air-core photonic bandgap fibers", Optic Express, Vol. 11, No. 23, pp. 3100-3109, 2003.

[27] S. chen and L. Shen, "Ultrahigh Birefringent Photonic Crystal Fiber with Ultralow Confinement Loss”, IEEE Photonic technology letters, Vol. 19, No. 4, pp. 185-187, 2007.

[28] C. Mao, B. Huang, Y. Wang, Y. Huang, L. Zhang,Y. Shao and Y.I. Wang, "High-sensitivity gas pressure sensor based on hollow-core photonic bandgap fiber Mach-Zehnder interferometer", Opt Exp, Vol. 26, No. 23, pp 30108-30116, 2018.

[29] 0. Svelto, Principles of Lasers, fifth ed., Chapter 4, Springer, Boston, 2010.

[30] F. Yang, W. Jin, Y. Cao, H. Ho, Y. Wang," Towards high sensitivity gas detection with hollow-core photonic bandgap fibers", Opt. Express, vol. 22, vol. 11, pp. 24894-24907, 2014.

[31] A.M. Cubillas, M. Silva-Lopez, J.M. Lazaro, O.M. Conde, M.N. Petrovich, J.M. Lopez-Higuera, Methane detection at 1670-nm band using a hollow-core photonic bandgap fiber and a multiline algorithm, Opt. Express, vol. 15, No. 26, pp. 17570-17576, 2007. 
[32] K.Z. Aghaie, M.J.F. Digonnet, S. Fan, Experimental assessment of the accuracy of an advanced photonic-bandgap-fiber model, J. Lightw. Technol, vol. 31, No. 7, pp.1015-1022, 2013.

[33] L. Filipovic and S. Selberherr, "Thermo-Electro-Mechanical simulation of semiconductor metal oxide gas sensors", MDPI Journal of Materials, vol. 12, pp. 1-37, 2019.

[34] MdI. Islam, K. Ahmed, S. Sen, S. Chowdhury, B.K. Paul, MdS. Islam, M.B.A. Miah, S. Asaduzzaman, "Design and optimization of photonic crystal fiber-based sensor for gas condensate and air pollution monitoring", Photon Sens, Vol. 7, No. 3, pp. 234-245, 2017.

[35] K. Saitoh, N.J. Florous, T. Murao, and M. Koshiba, "Design of photonic band gap fibers with suppressed higher-order modes: Towards the development of effectively single mode large hollow-core fiber platforms," Opt. Express, vol. 14, pp. 7342-7352, 2006.

[36] L. Li, Q. Chen, Q. Yuan, C. Liang and K. Sawaya, "Surface-wave suppression band gap and planewave reflection phase band of mushroom-like photonic band gap structures", Journal of Applied Physics, Vol. 19, No. 4, pp. 185-187, 2007.

[37] Y. L. Hoo, W. Jin, L. Xiao, J. Ju, and H. L. Ho, "Highly sensitive photonic crystal based absorption spectroscopy," Sens. Actuat, B. 145, pp. 110-113, 2010. 


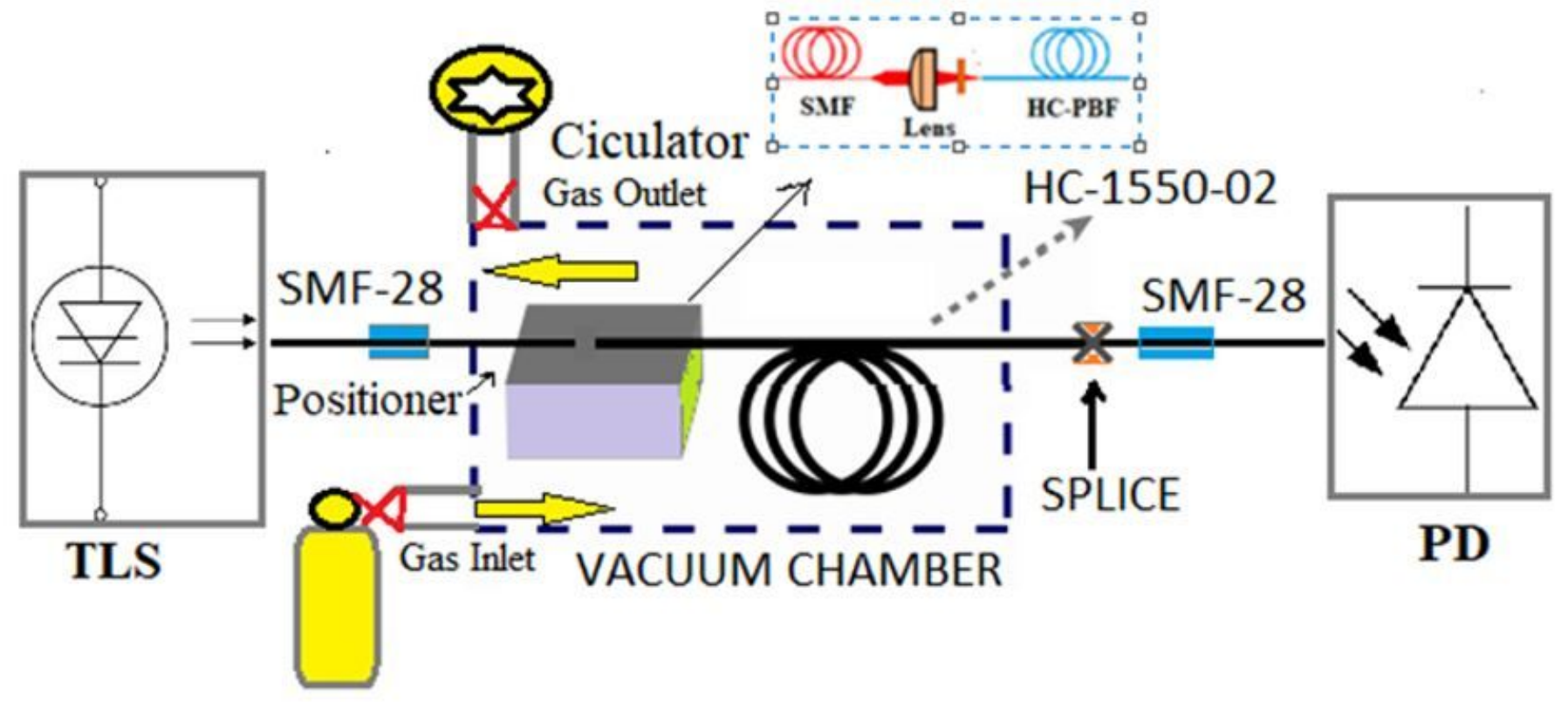

Figure 1

The schematic diagram of the of the proposed HC-PBGF based gas sensor.

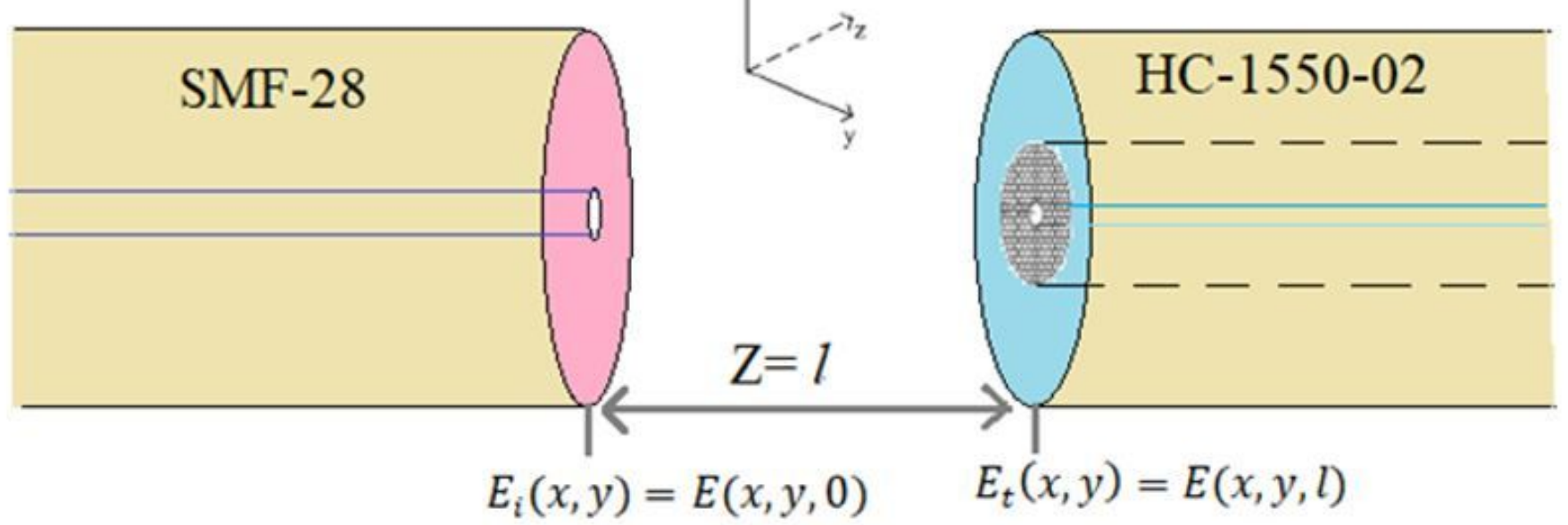

Figure 2

Schematic of the optical mode excitation from SMF-28 to HC-1550-02 fiber. 


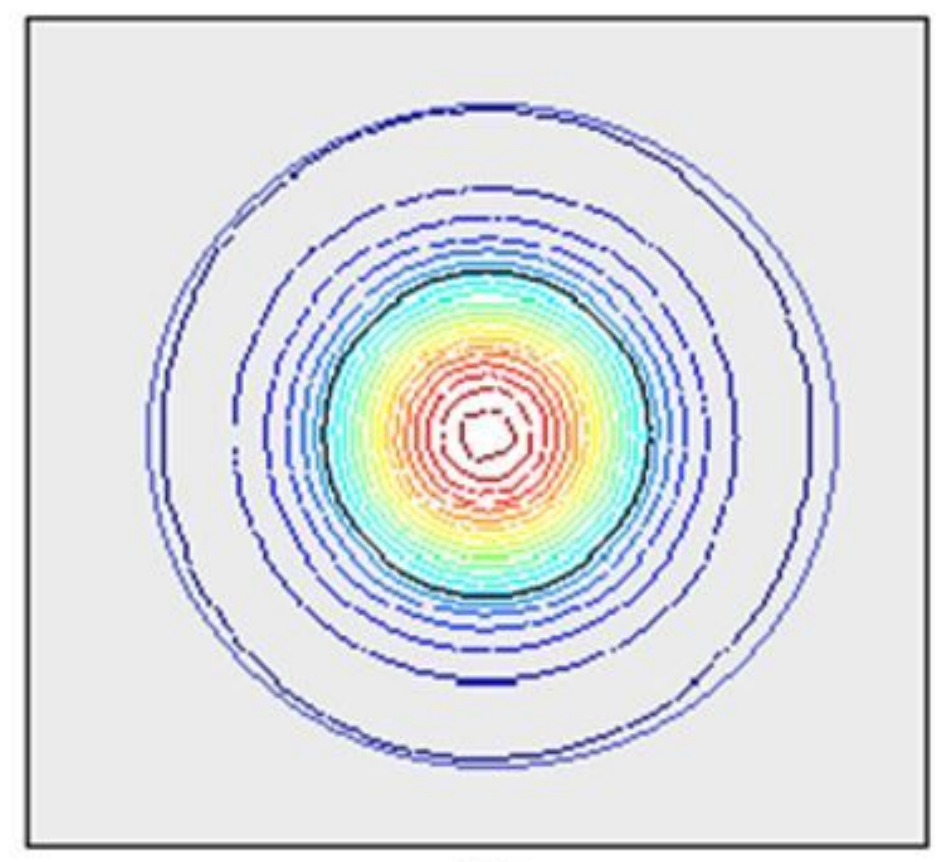

(a)

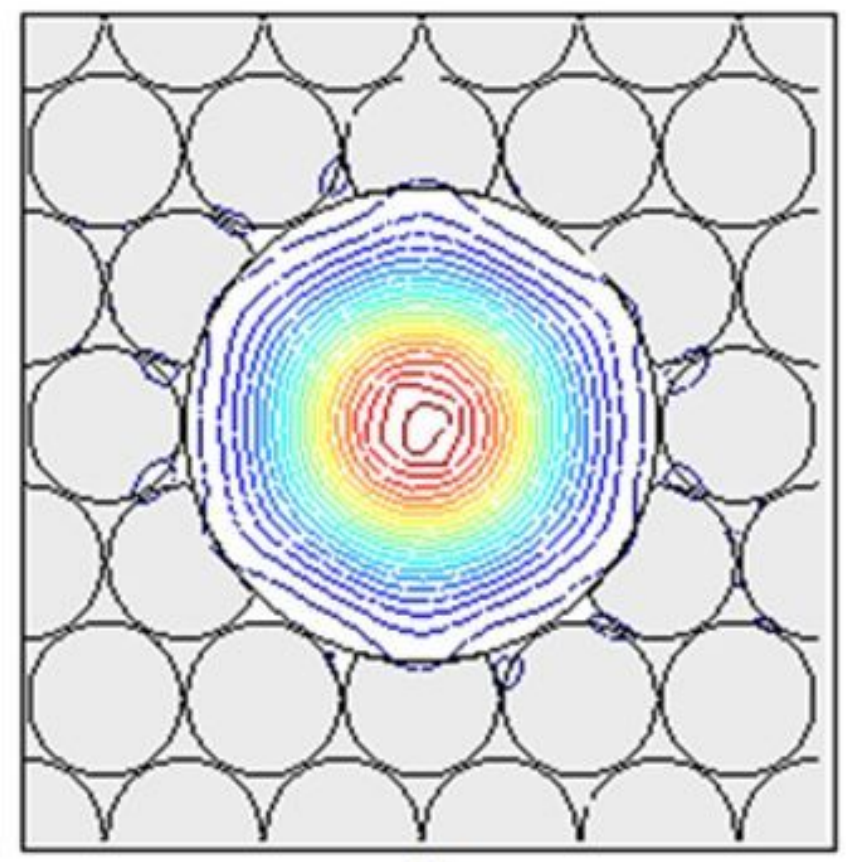

(b)

\section{Figure 3}

The representation of fundamental mode distribution for (a) single mode fiber and (b) hollow-core photonic bandgap fiber. 


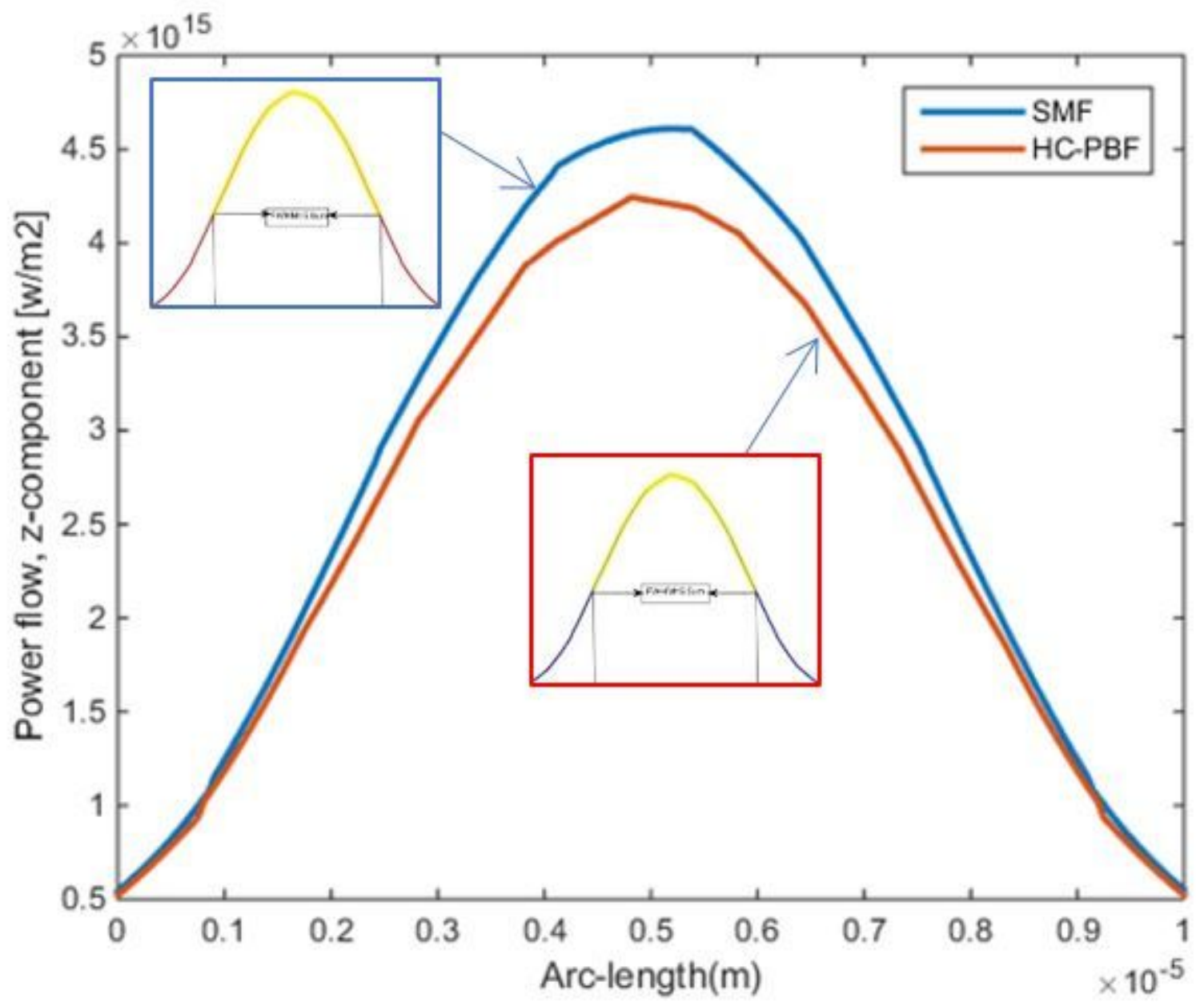

Figure 4

The normalized electric field intensities versus the core radius. 


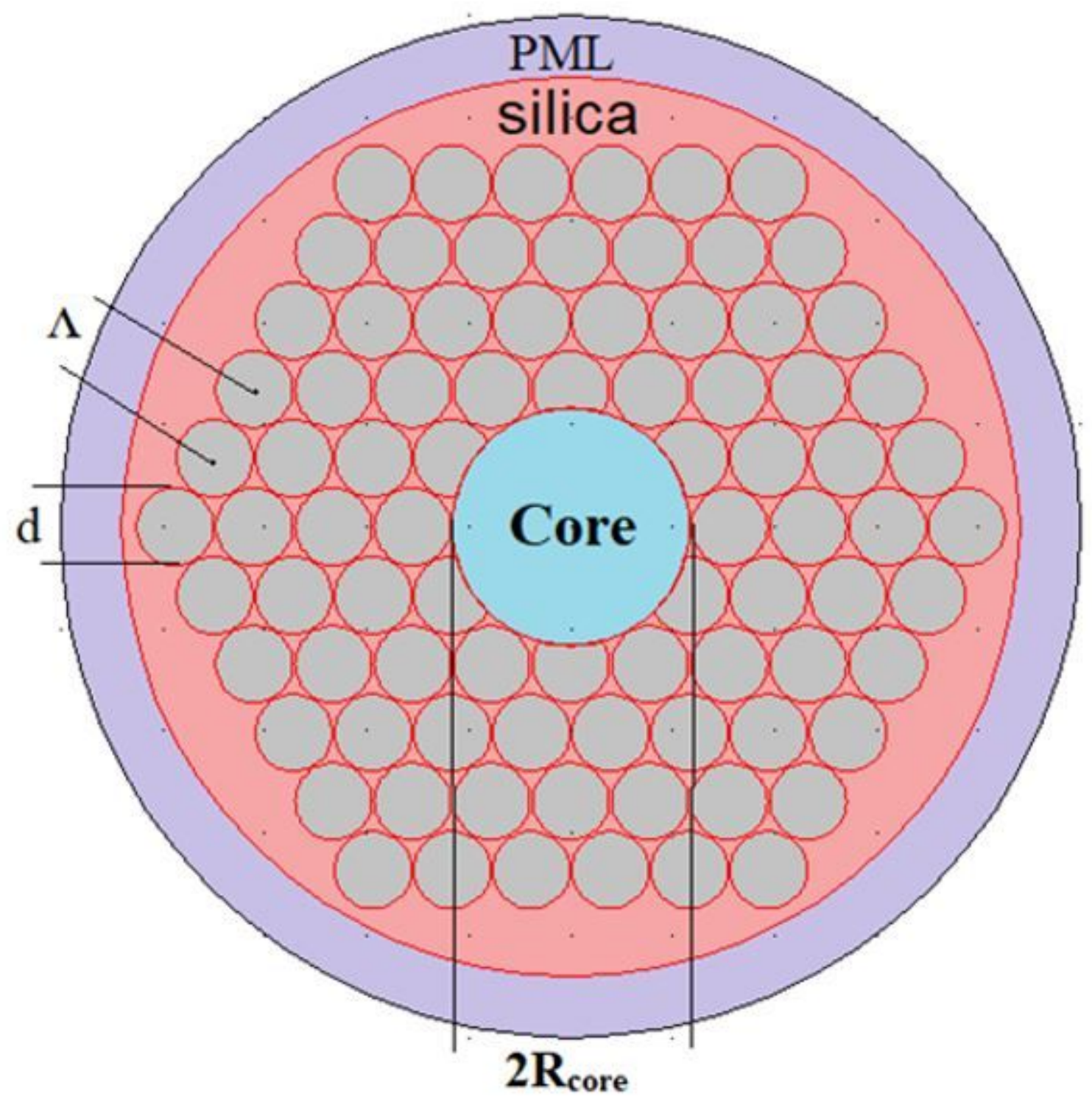

Figure 5

The schematic diagram of the hollow-core photonic bandgap fiber (NKT Photonics, HC-1550-02). 


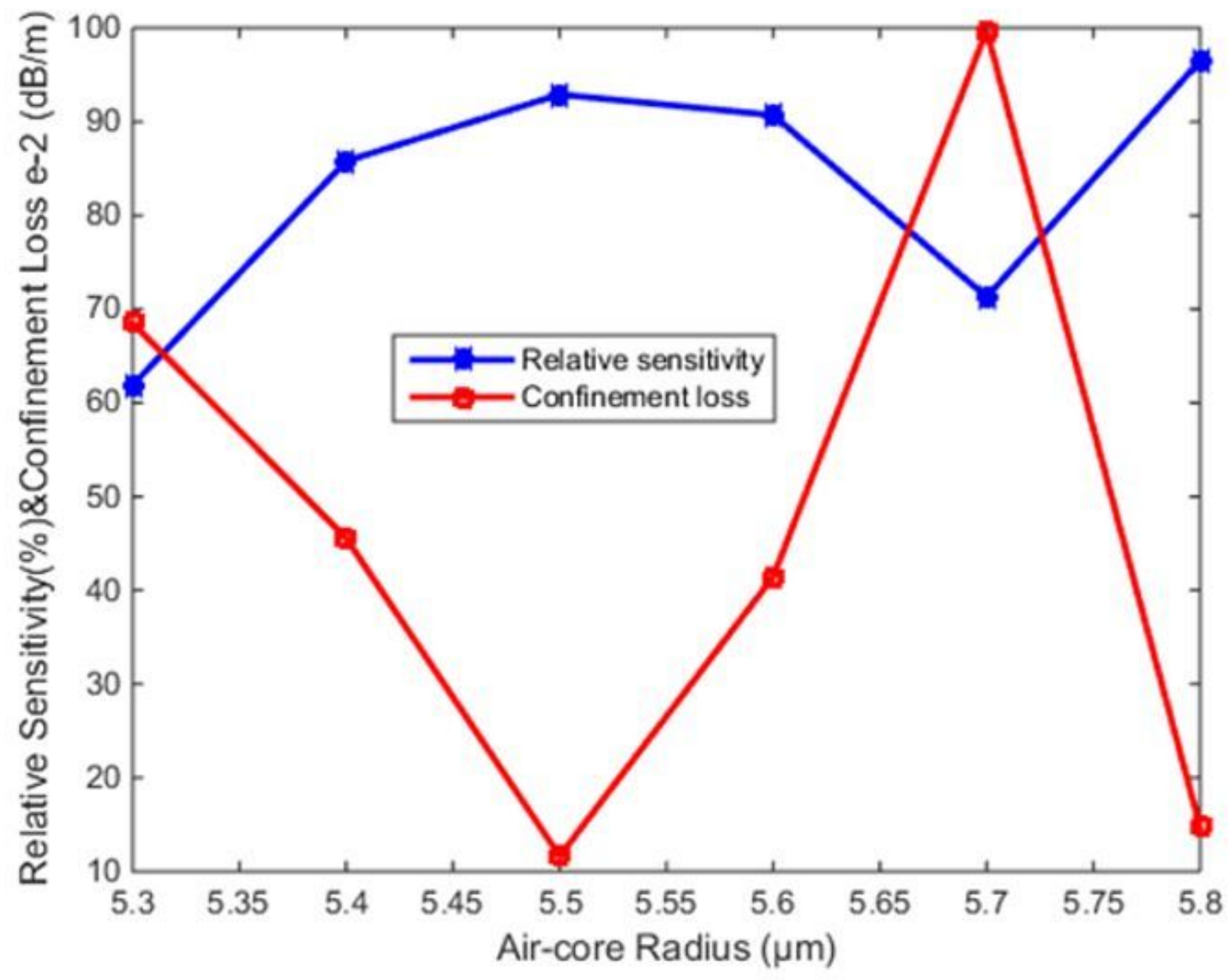

Figure 6

The relative sensitivity and confinement loss versus hollow-core radius. 


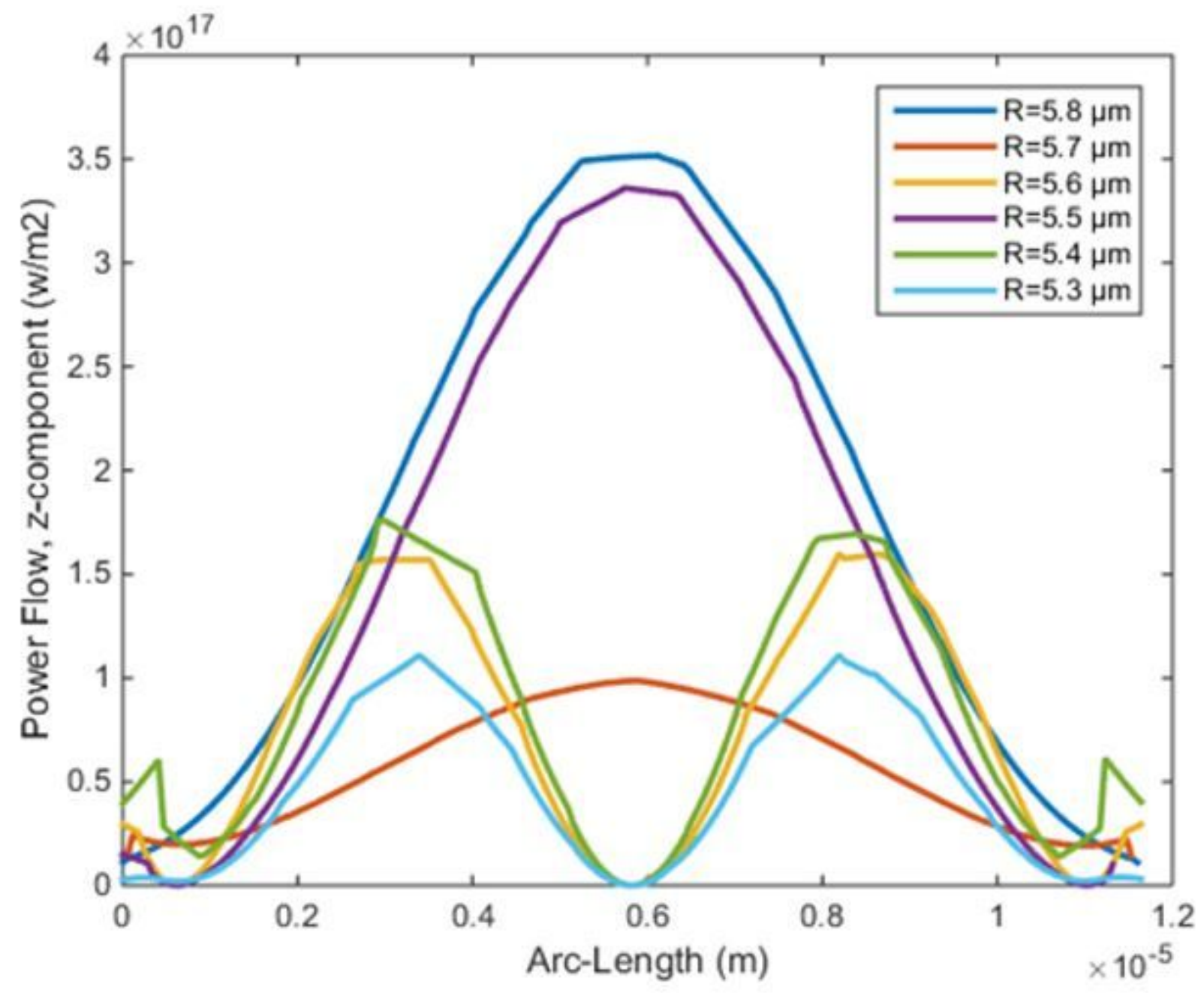

Figure 7

The relative sensitivity and confinement loss versus hollow-core radius. 